\title{
LA INNOVACIÓN
}

\section{EN EMPRESAS DE LOS SECTORES INDUSTRIAL Y DE Servicios: Caso ciudad de Chihuahua, México}

\author{
Juan Óscar Ollivier Fierro \\ Héctor Martínez Lara \\ José Alfonso Álvarez Terrazas \\ Universidad Autónoma de \\ Chihuahua
}

RECIBIDO: 2 DE MAYO DE 2017 Aceptado: 17 de agosto de 2017

\section{RESUMEN}

1 análisis detallado de
las innovaciones en las
empresas revela que tiene múltiples aspectos qué considerar, como es el grado de tecnología tangible e intangible en ellas. El objetivo del presente estudio es analizar las diferencias en los procesos de innovación de las empresas en los sectores de servicios y de la industria. El método se basó en una encuesta aleatoria a 116 empresas pequeñas y medianas, aplicando el instrumento europeo cis (Com- munity Innovation Survey) en la ciudad de Chihuahua, México. Los resultados muestran las principales características de la innovación tecnológica, orientada preferentemente al sector industrial y la no tecnológica, orientada a ambos sectores. Se encontraron diferencias entre sectores, la mayor parte favorables al sector industrial, en los cuatro tipos de innovación reconocidos por las instituciones internacionales, las inversiones en investigación y desarrollo, contratada y la comprada, los registros de propiedad inte- 
lectual y las alianzas con otras organizaciones. En general, este estudio aporta elementos que permiten una mayor comprensión sobre las diferencias en el proceso de innovación de las empresas en los sectores de servicios de la industria en un país en desarrollo.

Palabras clave: Innovación, tecnología, sectores económicos.

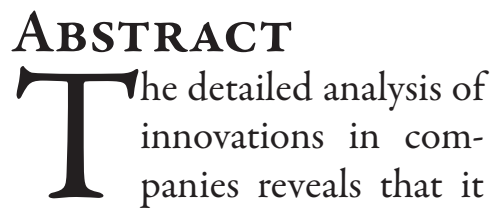
has multiple aspects to consider, such as the degree of tangible and intangible technology in them. The objective of the present study is to analyze the differences in the innovation processes of companies in the services and industry sectors. The method was based on a random survey of 116 small and medium-sized enterprises using the European Community Innovation Survey (cis) instrument in the city of Chihuahua, Mexico. The results show the main characteristics of the technological innovation oriented preferably to the industrial sector and the non-technological oriented to both sectors. Differences were found between sectors most favorable to the industrial sector, in the four types of innovation recognized by international institutions, investments in research and development, contracted and purchased, intellectual property registries and alliances with other organizations. As a general conclusion, this study provides elements that allow a better understanding of the differences in the innovation process of companies in the services and industry sectors in a developing country.

Keywords: Innovation, technology, economic sectors.

\section{INTRODUCCIÓN}

nte las fuerzas econó-
micas de la globaliza-
ción y el vertiginoso avance de las tecnologías de información y comunicaciones, la innovación se ha convertido en una de las más importantes estrategias que han seguido las empresas para sobrevivir y crecer en un ambiente de fuerte competencia local e internacional (Von Hippel, 2005), particularmente en los países con sistemas neolibera- les basados en economías de mercado cada vez más abiertas (Friedman, 2005), como es el caso de México.

A pesar de la importancia de la innovación, su percepción, conceptos, instrumentos de medición y en general, la literatura sobre ella en las empresas, se encuentra en buena medida orientada hacia la innovación tecnológica que se desarrolla en las empresas del sector industrial o de manufactura, restando importancia a la innovación no tecnológica y la que se lleva a cabo en las empresas del sector de servicios. Esta situación es paradójica puesto que el sector terciario o de servicios, no solo representa más de dos tercios del pib (Producto Interno Bruto), sino que es el sector con mayor crecimiento, particularmente en los países desarrollados (Carrasco, 2015), denominados anacrónicamente "industrializados", dada esta tendencia creciente hacia la terciarización de sus economías.

El esquema tradicional del proceso de innovación en las empresas manufactureras, generalmente está basado en la innovación tecnológica en los productos que se genera en las 
áreas de investigación y desarrollo $(\mathrm{I}+\mathrm{D})$, que posteriormente al ser introducidos a los mercados se convierten en innovaciones (i), cuyas ventas deben proporcionar los retornos de las inversiones efectuadas en I+D (Drucker, 1984), de ahí que comúnmente este proceso completo se representa con las siglas $\mathrm{I}+\mathrm{D}+\mathrm{i}$.

Además de estas innovaciones en los productos, se encuentran las innovaciones en los procesos, cuya principal diferencia es que normalmente no se introducen en los mercados (salvo la venta de licencias de fabricación o Know How), sino que más bien están orientadas a mejorar los procesos de producción de la propia empresa, como puede ser para incrementar su eficiencia o productividad y/o la calidad de los productos para incrementar su competitividad. Posteriormente se suma a estos dos tipos de innovación, las realizadas en la mercadotecnia y en la organización, de tal forma que estos cuatro tipos de innovación: producto, proceso, mercadotecnia, y organización son los reconocidos por el Manual de Oslo de la Organización para la Cooperación y el Desarrollo Económico (Eurostat, 2005).

En relación con esta temática, el problema de investigación detectado consiste en la escasa información en la literatura sobre los procesos de innovación en empresas pequeñas y medianas en países en desarrollo, particularmente las diferencias en las innovaciones entre las empresas de los sectores económicos de servicios y de la industria (Jacobsson y Bergek, 2006; Jasso y Torres, 2012).

El objetivo general de esta investigación es presentar un panorama general de la situación actual en cuanto a las principales características de los procesos de innovación en las empresas pequeñas y medianas chihuahuenses, distinguiendo el sector industrial y el de servicios, así como las principales relaciones entre las variables de interés de las empresas en ambos sectores económicos.

El presente trabajo se divide en las siguientes partes: 1) una introducción sobre la temática, el problema y objetivo, 2) una breve presentación de las principales obras de la literatura relativa, 3) una des- cripción del tipo y método de investigación seguido, 4) la presentación de los resultados descriptivos e inferenciales sobre las variables de interés, 5) como conclusión se presentan los principales hallazgos, y 6) las referencias bibliográficas.

\section{Marco TEÓRICO}

lcuerpo teórico sobre la
innovación en la época
moderna nace prácticamente en el seno de la teoría evolucionista relativa al cambio tecnológico con la obra de Schumpeter (1934), que acuña el concepto de innovación el cual ha prácticamente subsistido hasta la fecha tal como se le reconoce en la literatura y particularmente por las instituciones internacionales, como es el caso de la ocde (2011), que ha establecido estándares sobre este tema. El elemento central de este concepto de innovación es el de reconocer al invento o producto mejorado como tal, una vez que se encuentra comercializado en el mercado, constituyéndose en un factor del desarrollo económico, siendo el empresario el agente innovador. Esta teoría económica estudia el proceso de ge- 
neración y difusión de las tecnologías, sus relaciones con los cambios de las industrias y de los mercados, considerando los principios del desequilibrio, de la competencia y los ciclos económicos (Schumpeter, 1934).

Posteriormente el británico Freeman (1975), dentro de la escuela neo-schumpeteriana estudia la estrecha relación entre las innovaciones y los cambios económicos en contextos a nivel de país, introduciendo el concepto de Sistema Nacional de Innovación (sni) con la colaboración del sueco Lundvall, haciendo referencia a los ciclos económicos de Kondratieff.

Cabe destacar que la innovación de productos y procesos fue considerada durante la casi totalidad del siglo $\mathrm{xx}$, propia de las grandes corporaciones, sin embargo, actualmente en el contexto de la globalización y las tic se ha extendido a medianas y pequeñas empresas que han mostrado aportaciones significativas en el campo de las innovaciones (Rothwell y Zegveld, 1982; Pavitt et al. 1987; Ollivier y Thompson, 2009).

En la parte final del siglo pasado, una de las obras más importantes de la literatura sobre la innovación es la del británico Keith Pavitt (1984), en la que resume una investigación basada en dos mil innovaciones realizadas por empresas británicas a partir de la cuarta década del siglo pasado, resultando de ella su conocida taxonomía de las empresas que innovan y determinan el cambio tecnológico, que las clasifica en orden creciente a su capacidad innovadora endógena en la siguiente forma.

1) Empresas dominadas por los proveedores.

2) Empresas de producción intensiva.

3) Empresas basadas en ciencia.

En cuanto al papel de la tecnología, la primera categoría serían empresas usuarias, la tercera productoras y en la segunda se encuentran ambas, usuarias y productoras (Pavitt, 1984).

Posteriormente los autores Miozzo y Soete (2001), en su estudio sobre las empresas de servicio agregan a la taxonomía de Pavitt, la categoría de empresas basadas en redes, con dos subgrupos: las intensivas en escala basadas en redes físicas como son las de transpor- tes y ventas al mayoreo y las empresas basadas en redes de información, como son las de finanzas, seguros y comunicaciones. Cabe destacar que, en materia de innovación, son cada vez de mayor importancia las fuentes de información, de cooperación y alianzas con otras organizaciones, evidenciándose que las empresas con mayor innovación son también las que cuentan con mayores alianzas con diversas organizaciones (Gobble, 2010).

Esta estrategia de interrelaciones, ha generado algunos modelos, como el Triángulo de Sábato (Casas, 2004) y el conocido de la Triple hélice, propuesto por Leydesdorff y Etzkowitz (1998), basado en la interacción de manera coordinada de los tres componentes de la hélice: academia, industria y gobierno, coordinados por el último, con el objetivo esencial del fomento de la ciencia y la tecnología para generar la innovación y su consecuente desarrollo económico.

En relación con la distinción entre innovaciones tecnológicas y no tecnológicas en las empresas, se observa más que sustitutas son complementarias, un ejemplo de ello es 
cuando una empresa adquiere una maquinaria; requiere entrenamiento a su personal para su operación, tanto en empresas manufactureras como de servicios. Pudiendo concluir que la innovación se encuentra en buena medida en función de la complementariedad entre actividades tecnológicas y no tecnológicas (Carrasco, 2015). Igualmente se ha encontrado que los principales determinantes de las innovaciones tecnológicas y no tecnológicas en ambos sectores económicos son: i) los niveles de inversión en ellas; y ii) el tamaño de la empresa. Ambos tipos de innovaciones están positivamente asociados a ganancias en productividad en el sector de servicios pero de manera más importante, las no tecnológicas. Contrariamente sucede en el sector de manufactura en donde las innovaciones tecnológicas influyen de mayor manera en la productividad (Aboal y Garda, 2016).

En cuanto a la complementariedad de innovaciones tecnológicas y no tecnológicas, se observa una tendencia en la estrategia de las empresas manufactureras a incrementar la innovación en los servicios asociados a sus productos, ya sea por la adopción de nuevos servicios o por la extensión de la cobertura de los existentes (Visnjick, Van Looy y Neely, 2013).

Por otra parte, la innovación en el sector de servicios, generalmente de tipo no tecnológica o más propiamente menos intensiva en tecnología, orienta en buena medida la cultura de la empresa al definir sus valores para incrementar el interés del cliente a través de la experiencia, la cual se convierte en el objeto de la transacción adicionalmente a la compra de los bienes físicos en el sector del comercio. Existe un interés renovado, en como por medio de actividades creativas, las compañías de servicios pueden emplear el paradigma de la experiencia del cliente para crear ventaja competitiva (D'Ippolito y Timpano, 2016).

A pesar de que las innovaciones tecnológicas han estado tradicionalmente asociadas a las actividades de $\mathrm{I}+\mathrm{D}$, estas adquieren una cada vez mayor importancia en las innovaciones no tecnológicas, lo cual lleva a decir que las inversio- nes en actividades de $\mathrm{I}+\mathrm{D}$, demuestran sus efectos y rentabilidad tanto en empresas del sector manufacturero como el de servicios. Igualmente existe una asociación positiva entre la amplitud de las fuentes de conocimiento y el éxito en innovaciones en general (Leiponen, 2012).

Referente a las fuentes de conocimiento, surge el paradigma de la innovación abierta (del inglés open innovation), que postula que en nuestro mundo actual donde el conocimiento es asequible y globalmente difundido, particularmente a través de la red del internet, las organizaciones tienen la posibilidad de acceder y conocer al conocimiento generado en otras organizaciones, como pueden ser clientes, proveedores o competidores, el cual, sumado a sus propias fuentes, puede enriquecer y complementar su proceso de innovación (Chesbrough, 2003, Gianiodis et al., 2014).

Vale la pena señalar que esta estrategia de la innovación abierta implica una nueva cultura en la empresa que debe incluir un mayor énfasis en los procesos de la propiedad intelectual, que pueden surgir de 
los esquemas de cooperación y alianzas (Escorsa y Valls, 2009).

\section{Metodología}

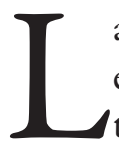

a escasa información encontrada en la literatura sobre los procesos de innovación en empresas pequeñas y medianas en países en desarrollo, particularmente las diferencias en las innovaciones entre las empresas de los sectores económicos de servicios y de la industria (Jacobsson y Bergek, 2006, Jasso y Torres. 2012), motivó al desarrollo de esta investigación.

Será interesante integrar información sobre las principales diferencias entre los procesos de innovación en las empresas de los sectores industrial y de servicios, la cual podrá ser útil para el diseño de políticas públicas de fomento a las innovaciones diferenciadas en cada uno de estos sectores (Santiago, De Fuentes y Dutrenit, 2017), así como a las mismas empresas para el diseño de estrategias orientadas a la innovación de sus productos para incrementar su competitividad.

Las preguntas de investigación referentes a las empresas pequeñas y medianas de la ciudad de Chihuahua, fueron las siguientes.

1) ¿Cuáles son las principales características de los procesos de innovación distinguiendo el sector de servicios y de la industria?

2) ¿Cuáles son las principales relaciones entre las variables de interés que intervienen en los procesos de innovación, distinguiendo el sector de servicios y de la industria?

El objetivo general es presentar un panorama general de la situación actual en cuanto a las principales características de los procesos de innovación en las empresas pequeñas y medianas chihuahuenses, distinguiendo el sector industrial y el de servicios, así como las principales relaciones entre las variables de interés de las empresas en ambos sectores económicos.

\section{Los OBJETIVOS ESPECÍFICOS FUERON:}
1) Identificar las principales características de los proce-

sos de innovación, distinguiendo el sector de servicios y de la industria.

2) Analizar las principales relaciones entre las variables de interés que intervienen en los procesos de innovación distinguiendo el sector de servicios y de la industria.

\section{LA HIPÓTESIS CENTRAL VA EN EL SENTIDO QUE:}

$\mathrm{H}$ parte de sus innovaciones son de tipo tecnológico, requieren una mayor inversión en I+D, registro de patentes y alianzas que las empresas en el sector de servicios cuya mayor parte de sus innovaciones son de tipo no tecnológico.

Se desarrolló un estudio empírico, basado en una encuesta a 116 empresas, con un enfoque esencialmente cuantitativo, de tipo no experimental, transversal en el año 2016, en la ciudad de Chihuahua y su zona de influencia (Cuauhtémoc y Delicias). Los sujetos de estudio fueron empresas en los estratos pequeñas 
Cuadro 1. Empresas de la muestra según su sector económico y tamaño.

\begin{tabular}{cccc}
\hline & \multicolumn{3}{c}{ Tamaño } \\
\hline Sector económico & Pequeñas & Medianas & Total \\
\hline Servicios & 38 & 7 & 45 \\
\hline Industria & 59 & 12 & 71 \\
Suma & 97 & 19 & 116 \\
\hline
\end{tabular}

Fuente: Elaboración propia.

y medianas de los sectores, comercio, servicios, industria manufacturera y construcción de la ciudad de Chihuahua, México. Cabe señalar que en el grupo del sector de servicios se consideró el comercio y la industria de construcción.

La población de empresas constituidas es del orden de 14 000 en la ciudad de Chihuahua y su zona de influencia. Para el cálculo del tamaño de esta muestra se consideró una distribución binomial, con una proporción de empresas que cuentan con un proceso de innovación en cualesquiera de sus cuatro tipos, de $50 \%$ (p $=0.5$, caso crítico), con un 10 $\%$ de error y $95 \%$ de confianza (empleando la fórmula $n$ $=p \cdot q \cdot Z 2 / E 2)$, resultando un tamaño calculado $n$ de 96 empresas. A continuación en el cuadro 1 se presenta la muestra de 116 empresas, es decir, 20 adicionales al mínimo para incrementar su representativi- dad, levantada en la encuesta hasta el mes de diciembre de 2016, de acuerdo con su tamaño y sector económico.

En el caso de las ciudades del norte de este país, donde se encuentra Chihuahua, se ha desarrollado, dada su cercanía geográfica, una economía en buena medida dependiente de la economía de Estados Unidos de América (eua), tanto en sus importaciones como en sus exportaciones. En estas últimas se incluye la actividad de las empresas maquiladoras de creciente importancia, las cuales aun cuando su capital de origen puede ser de diferentes países, tienen como denominador común que el mercado final de sus productos es el de eua. La presencia de esta industria maquiladora de exportación (ime) en los estados fronterizos norteños ha generado una gran cantidad de empresas, tanto en el sector de manufactura, como en el de servicios (Ollivier, 2007).

Se realizó la encuesta aplicando como cuestionario el instrumento cis (Community Innovation Survey), en su última versión 2012, desarrollado y empleado por la Unión Europea para la medición de variables relacionadas a la innovación en las empresas. Los cuestionarios se llenaron en entrevistas cara a cara con los responsables de las empresas, llevadas a cabo por maestros y estudiantes de posgrado de la Universidad Autónoma de Chihuahua. Cabe señalar, que este cuestionario está diseñado para levantar información sobre innovación en las empresas en los últimos tres años.

Las variables de interés principales consideradas en el estudio a nivel de empresas, distinguiendo su sector económico, fueron: 
- Innovación en productos

- Innovación en procesos

- Innovación en mercadotecnia

- Innovación en la organización

- Fuentes de información para la innovación

- Alianzas con organizaciones para la innovación

- Obstáculos para la innovación

- Registro de propiedad intelectual

- Inversiones en investigación y desarrollo

- Efectos de la innovación

La estrategia de análisis consistió primeramente en un análisis descriptivo de las principales variables asociadas a la innovación y posteriormente un análisis inferencial a través de la prueba de dependencia de Chi cuadrada dada la naturaleza nominal y ordinal de los niveles de medición de las variables de interés, con el apoyo del paquete estadístico SPSS v20.

El alcance de los resultados del estudio es su generalización a la ciudad de Chihuahua, Delicias y Cuauhtémoc. $\mathrm{Su}$ principal limitación es la veracidad de las respuestas a los 116 cuestionarios válidos de la muestra. Cabe señalar que no se consideraron 23 cuestionarios debido a la incongruencia de la información proporcionada.

La confiabilidad de la encuesta medida por el Alfa de Cronbach fue de 0.76 para los 74 reactivos (ítems) considerados.

\section{ANÁlisis E INTERPRETACIÓN DE RESULTADOS}

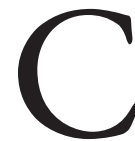

omo datos generales, se tuvieron en el sector de servicios una medias aritméticas en las ventas anuales de 22761356 pesos y de 26 trabajadores en el sector de la industria, estos mismos datos fueron 12908 726 y 34 trabajadores.

\section{Resultados DESCRIPTIVOS}

$1 \begin{aligned} & \text { continuación se mues- } \\ & \text { tran los resultados } \\ & \text { descriptivos de las }\end{aligned}$ principales variables relacionadas con la innovación en sus cuatro tipos. En relación con la innovación en los productos, los resultados encontrados se muestran en el cuadro 2 .
Se observa que es ligeramente mayor el porcentaje de empresas que realizan innovación en los productos en el sector de la industria que en el de servicios. Estos porcentajes de 31.8 y $38 \%$, se reducen a 24.2 y $34.3 \%$, respectivamente, cuando se especifica que las innovaciones son nuevas para su mercado. En relación con la innovación en los procesos, los resultados encontrados se muestran en el cuadro 3.

En congruencia con la innovación en productos, se observa que es ligeramente mayor el porcentaje de empresas que realizan innovación en los procesos en el sector de la industria $(76.1 \%)$ que en el de servicios (65.9 \%). En relación con la innovación en la mercadotecnia, los resultados encontrados se muestran en el cuadro 4.

Se observa que de la misma forma que las innovaciones en productos y en procesos, también se tiene un mayor porcentaje de empresas que han realizado innovaciones en mercadotecnia en el sector industrial que en el de servicios en ambas variables. Cabe señalar que en relación con la variable de distribución, logística y venta, las 
Cuadro 2. Empresas que han realizado innovaciones en sus productos, según su sector económico (\%).

\begin{tabular}{cccc}
\hline & \multicolumn{4}{c}{ Innovaciones en sus productos } & Total \\
\hline Sector económico & No & Sí & 100 \\
\hline Servicios & 68.2 & 31.8 & 100 \\
\hline Industria & 62.0 & 38.0 & 100 \\
\hline Total & 64.3 & 35.7 & \\
\hline
\end{tabular}

Fuente: Elaboración propia.

Cuadro 3. Empresas que han realizado innovaciones en sus procesos en los últimos tres años según su sector económico (\%).

\begin{tabular}{cccc}
\hline & \multicolumn{2}{c}{ Innovaciones en sus procesos } & Total \\
\hline Sector económico & No & Sí & 100 \\
\hline Servicios & 34.1 & 65.9 & 100 \\
\hline Industria & 23.9 & 76.1 & 100 \\
Total & 27.8 & 72.2 & \\
\hline
\end{tabular}

Fuente: Elaboración propia.

Cuadro 4. Empresas que han realizado innovaciones en mercadotecnia, según su sector económico (\%).

\begin{tabular}{ccc}
\hline & \multicolumn{2}{c}{ Innovaciones en mercadotecnia } \\
\hline Sector económico & En el diseño del producto o servicio & En la distribución, logística y venta \\
\hline Servicios & 47.7 & 22.7 \\
\hline Industria & 60.6 & 38.0 \\
Total & 55.7 & 32.2 \\
\hline
\end{tabular}

Fuente: Elaboración propia.

Cuadro 5. Empresas que han realizado innovaciones en la organización, según su sector económico (\%).

\begin{tabular}{cccc}
\hline & \multicolumn{3}{c}{ Innovaciones en la organización } \\
\hline Sector económico & $\begin{array}{c}\text { En gestión de la informa- } \\
\text { ción }\end{array}$ & $\begin{array}{c}\text { Cambios a nivel } \\
\text { de la organización }\end{array}$ & $\begin{array}{c}\text { En su relación con otras } \\
\text { empresas }\end{array}$ \\
\hline Servicios & 68.2 & 61.4 & 41.9 \\
\hline Industria & 60.6 & 52.1 & 33.8 \\
Total & 63.5 & 55.7 & 36.8 \\
\hline
\end{tabular}

Fuente: Elaboración propia. 
Cuadro 6. Inversiones anuales en I+D interna, externa (contratada) para el desarrollo de innovaciones y compra de tecnología, según su sector económico (inversiones en pesos y esfuerzo en porcentaje de inversión sobre ventas).

\begin{tabular}{ccccccc}
\hline $\begin{array}{c}\text { Sector } \\
\text { económico }\end{array}$ & $\begin{array}{c}\text { Inversión en } \\
\text { I+D interna }\end{array}$ & $\begin{array}{c}\text { Esfuerzo rela- } \\
\text { tivo de I+D } \\
\text { interna }\end{array}$ & $\begin{array}{c}\text { Inversión en } \\
\text { I+D externa o } \\
\text { contratada }\end{array}$ & $\begin{array}{c}\text { Esfuerzo rela- } \\
\text { tivo de I+D } \\
\text { externa }\end{array}$ & $\begin{array}{c}\text { Inversión en } \\
\text { compra de } \\
\text { tecnología* }\end{array}$ & $\begin{array}{c}\text { Esfuerzo rela- } \\
\text { tivo de compra } \\
\text { de tecnología }\end{array}$ \\
\hline Servicios & 105929 & 1.85 & 63455 & 2.02 & 206194 & 1.52 \\
\hline Industria & 179500 & 2.64 & 101231 & 1.8 & 382553 & 2.79 \\
\hline Total & 150889 & 2.29 & 83917 & 1.9 & 296757 & 2.02 \\
\hline
\end{tabular}

Fuente: Elaboración propia.

$\left.{ }^{*}\right)$ : La compra de tecnologías se refiere a compra de patentes, licencias, Know How, etcétera.

empresas manifestaron que la mayor parte de las innovaciones son debido al uso de la red de internet que ha permitido una mayor eficiencia en estos procesos.

En relación con la innovación en la organización en su conjunto, los resultados encontrados se muestran en el cuadro 5 .

Se observa que contrariamente a las innovaciones en los tres casos precedentes, el mayor porcentaje de empresas que han realizado innovaciones están en el sector de servicios en las tres variables consideradas. Este resultado es congruente con el hecho de que las empresas de servicios se orientan más a las innovaciones no tecnológicas como es el caso de las nuevas formas de trabajo en el conjunto de la organización. Este resultado es congruente con los estudios sobre este tema en Francia (Dandurand, 2005).

En cuanto a la capacitación del personal para llevar a cabo innovaciones, se encontró que la mayor parte de las empresas la realizan, siendo ligeramente mayor $(73.2 \%)$ el caso de las empresas del sector industrial que el de servicios (70.5\%).

Referente a los apoyos financieros, se encontró que solo el $9.1 \%$ de las empresas del sector de servicios y el 5.6 $\%$ del sector industrial los reciben de instituciones regionales, sin embargo, de instituciones federales, es ligeramente mayor: el $15.9 \%$ de las empresas de servicios y el 9.9 $\%$ del sector industrial los reciben. Se observa que en ambos casos, tanto regional como federal, son las empresas de servicios que reciben un mayor apoyo financiero.

Con respecto a las inversiones en $\mathrm{I}+\mathrm{D}$, ya sea realizada internamente o contratada a otra organización y la adquisición de tecnología, el cuadro 6 muestra estas tres variables: las inversiones anuales en pesos y el esfuerzo relativo en $\mathrm{I}+\mathrm{D}$ interno, contratado o comprado en un porcentaje de las ventas.

Se observa que tanto las inversiones en $\mathrm{I}+\mathrm{D}$ como en compra de tecnología es considerablemente mayor en las empresas del sector industrial que en el de servicios, tal como se muestra en la figura 1. Este resultado pone de relieve que en las empresas del sector industrial donde la mayor parte de las innovaciones son tecnológicas, requieren mayores inversiones en I+D. Lo mismo es en el esfuerzo relativo en las inversiones en $\mathrm{I}+\mathrm{D}$ internas $\mathrm{y}$ la compra de tecnología, con la excepción del caso de la I+D contratada, que es ligeramente mayor en el caso de las empresas de servicios, lo cual se pue- 
Figura 1. Comparativo en montos inversión en tecnología e $\mathrm{I}+\mathrm{D}$, en pesos.

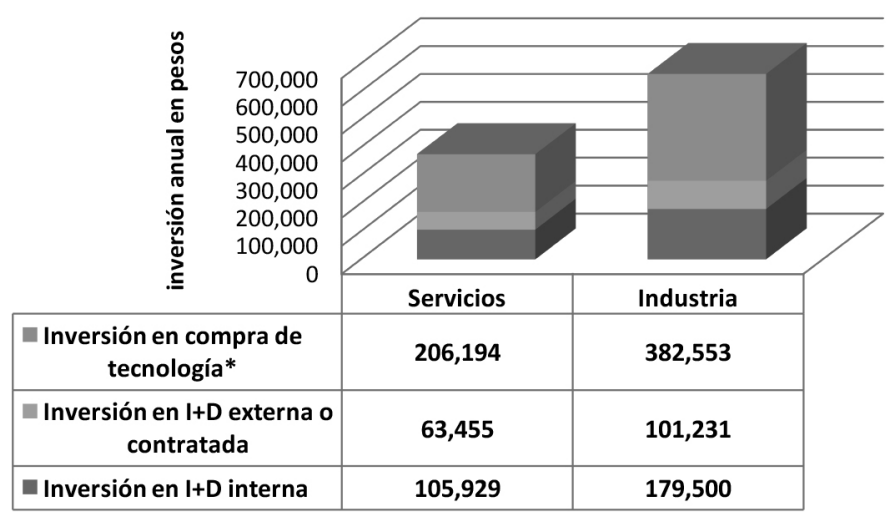

Fuente: Elaboración propia.

de deber a la ausencia de infraestructura de I+D propia. Este último resultado coincide con el estudio realizado por Teixeira y Becerra (2016), en el que encuentran que las fuentes científicas externas de información son cruciales para las empresas de servicios.

Relativo a las fuentes de información para realizar innovaciones, se encontró que la más importante fuente para las empresas del sector de servicios fueron los clientes ( 55.8 $\%)$, seguida de los proveedores $(52.3 \%)$, mientras que para las del sector industrial la más importante fueron los competidores (62\%), seguida de los proveedores (59.2\%). En el caso de la universidades, se encontró que es mayor en el caso del sector de la industria (32.4 $\%)$, que las empresas en el sector de servicios ( $25 \%$ ), como fuentes de información.

En cuanto a los obstáculos para realizar innovaciones, se encontró que el más importante para las empresas de ambos sectores fueron la falta de fondos, con $46.5 \%$ de las empresas de servicios y $55.6 \%$ de las industriales. En el caso de servicios, el segundo obstáculo es el costo de las innovaciones (40.9 \%) y en el caso de la industria el segundo es la falta de financiamiento con $3.4 \%$ de las empresas. Se observa que en todos los casos los principales obstáculos son de tipo económico.

Sobre los principales efectos de la innovación en las em- presas, la mejora en la calidad de los productos se encuentra en el primer lugar con el 79.5 $\%$ de las empresas de servicios y $80.3 \%$ de las del sector industrial. Igualmente en ambos sectores se encuentra en segundo lugar la penetración de nuevos mercado con 54.5 $\%$ de las empresas del sector de servicios y $59.2 \%$ del sector industrial.

\section{RESUlTADOS INFERENCIALES}

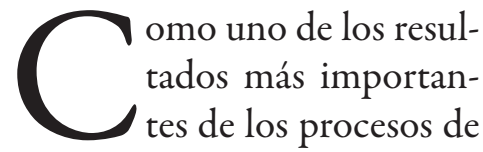
innovación están los registros de propiedad intelectual, los cuales se pueden registrar en México ya sea como propiedad industrial en el Instituto Mexicano de la Propiedad Industrial (impi), que incluyen patentes, diseños industriales y marcas entre otros o como derechos de autor a registrarse en el Instituto Nacional de Derechos de Autor (Indautor), tal como se muestra en la figura 2. 
Figura 2. Porcentaje de empresas con registros de propiedad intelectual, industrial o derechos de autor, según su sector económico (\%)

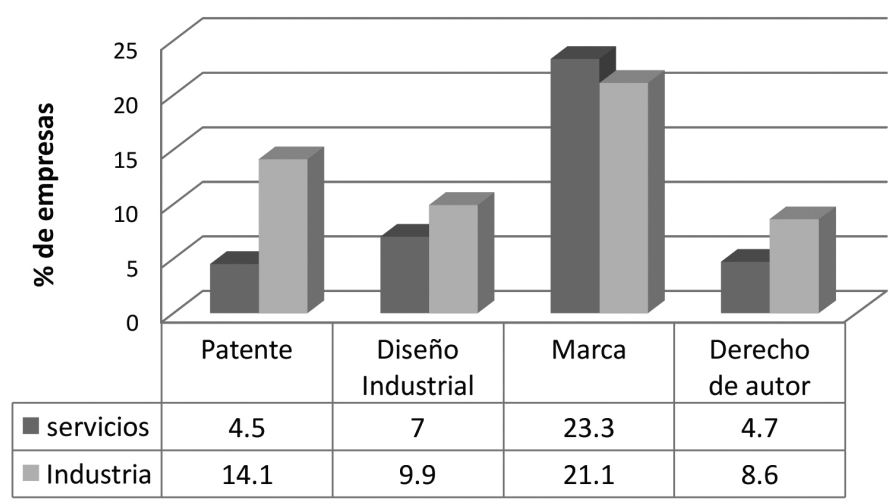

Fuente: Elaboración propia.

Se observa que el porcentaje de empresas industriales que han registrado patentes, diseños industriales y derechos de autor es mayor que el sector de servicios, salvo en el registro de marcas donde es ligeramente mayor el porcentaje de este último sector.

En el sector industrial se encontraron asociaciones significativas entre el registro de patentes y los otros tres registros de propiedad intelectual considerados, tal como se muestra en el cuadro 7 , donde los valores de la $\chi^{2}$ de la variable registro de patentes y su significancia estadística con las otras tres variables es de 47.37 $(\mathrm{P}=0.00)$ con el registro de diseños industriales; de 16.88
$(\mathrm{P}=0.00)$ con el registro de marcas; y $16.96(\mathrm{P}=0.00)$ con el registro de derechos de autor.

Este resultado muestra que aun cuando es relativamente bajo el porcentaje de empresas industriales que patentan (14.1\%), estas empresas tienen una cultura orientada hacia el registro de la propiedad intelectual, como es en los diseños industriales, marcas y derechos de autor.

Cabe observar que en el sector de servicios, cuyos valores se muestran en la figura 1 , no se encontraron estas asociaciones, lo cual pone de manifiesto la ausencia de una estrategia de registro de la propiedad intelectual en este sector.
Por otra parte, se encontró en el sector industrial una asociación significativa entre el registro de patentes y la realización de alianzas con otras organizaciones para la innovación, tal como se muestra en la siguiente tabla de contingencia (cuadro 8), entre estas variables, cuyo valor de la $\chi 2$ es de 4.39 con una significancia $\mathrm{P}=0.046$.

Este resultado sugiere que las alianzas favorecen el lograr el registro de patentes en este sector y viceversa.

Referente a las alianzas con otras organizaciones para la realización de innovaciones, se encontró una asociación significativa entre la realización de alianzas y el sector, que muestra que son las empresas de la industria las que emplean mayormente esta estrategia para la realización de innovaciones, tal como se muestra en el cuadro 9, con un valor de la $C h i$ cuadrada de 9.09 una significancia $\mathrm{P}=0.003$.

Referente a las organizaciones con las que se han establecido alianzas para la innovación, se encontró que en ambos sectores la principal ha sido con los clientes: 18.3 $\%$ de las empresas del sector 
Cuadro 7. Empresas del sector industrial que han registrado diseños industriales, marcas y derechos de autor, según su registro de patentes (\%).

\begin{tabular}{ccccccc}
\hline & \multicolumn{7}{c}{ Registros de propiedad intelectual } \\
\hline Registro de patentes & \multicolumn{2}{c}{ Diseño industrial } & \multicolumn{2}{c}{ Registro de marcas } & \multicolumn{2}{c}{ Derechos de autor } \\
\hline & No & Sí & No & Sí & No & Sí \\
\hline No & 00 & 0 & 86.9 & 13.1 & 96.7 & 3.3 \\
\hline Sí & 0.0 & 70.0 & 30.0 & 70.0 & 55.6 & 44.4 \\
Total & 90.1 & 9.9 & 78.9 & 21.1 & 91.4 & 8.6 \\
\hline
\end{tabular}

Fuente: Elaboración propia.

Cuadro 8. Tabla de contingencia que muestra el porcentaje de empresas del sector industrial que hacen alianzas con otras organizaciones para la realización de innovaciones y el registro de patentes (\%).

\begin{tabular}{cccc}
\hline & \multicolumn{3}{c}{ Registro de patentes } \\
\hline Alianza & No & Sí & Total \\
\hline No & 94.4 & 5.6 & 100 \\
\hline Sí & 77.1 & 22.9 & 100 \\
Total & 85.9 & 14.1 & 100 \\
\hline
\end{tabular}

Fuente: Del autor, con base en la encuesta.

Cuadro 9. Tabla de contingencia que muestra el porcentaje de empresas que hacen alianzas con otras organizaciones para la realización de innovaciones, para ambos sectores económicos (\%).

\begin{tabular}{cccc}
\hline & \multicolumn{3}{c}{ Hace alianzas } \\
\hline Sector económico & No & Sí & Total \\
\hline Servicios & 79.5 & 20.5 & 100 \\
\hline Industria & 50.7 & 49.3 & 100 \\
\hline Total & 61.7 & 38.3 & 100 \\
\hline
\end{tabular}

Fuente: Elaboración propia.

industrial y $9.1 \%$ del sector servicios, enseguida, en ambos sectores con los proveedores, $14.1 \%$ del sector industrial y $9.1 \%$ de servicios. Con las universidades se encuentran en cuarto lugar con $7 \%$ de las empresas industriales y $4.5 \%$ de servicios. Se encontró que es el sector industrial el que favorece estrategias de alianzas con las diferentes organizaciones para realizar innovaciones, particularmente con clientes y proveedores.

\section{Conclusiones}

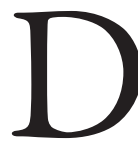
e los resultados anteriores, se pueden extraer las siguientes conclusiones sobre las principales diferencias en cuanto al 
proceso de innovación en las empresas de los sectores de servicios y de la industria. Los dos primeros puntos son relativos al primer objetivo específico, esencialmente descriptivo y el tercer es referente al segundo objetivo donde se exploran relaciones significativas entre las variables.

1) Se observa una mayor intensidad en las actividades relacionadas a las innovaciones en el sector de la industria, considerando los siguientes principales resultados.

a) Es ligeramente mayor el porcentaje de empresas que realizan innovación en los productos, en el sector de la industria (38\%) que en el de servicios (31.8\%).

b) Igualmente se encontró que es ligeramente mayor el porcentaje de empresas que realizan innovación en los procesos en el sector de la industria $(76.1 \%)$ que en el de servicios (65.9\%).

c) De la misma manera en las innovaciones en la mercadotecnia, también se tiene un mayor porcentaje de empresas que han realizado innovaciones en el sector industrial que en el de servicios, tanto en el diseño del producto (60.6/47.7 \%), como en la distribución, logística y venta (38/22.7\%).

d) Referente a las inversiones anuales en $\mathrm{I}+\mathrm{D}$ interna $\mathrm{y}$ contratada como en compra de tecnología es considerablemente mayor en las empresas del sector industrial ( $\$ 663$ 578), que en el de servicio (\$375 284), lo cual muestra que en las empresas del sector industrial donde la mayor parte de las innovaciones son tecnológicas, requieren mayores inversiones en I+D. Este resultado se refleja en el esfuerzo relativo en las inversiones en $\mathrm{I}+\mathrm{D}$ internas y la compra de tecnología, medido en inversión en $\mathrm{I}+\mathrm{D}$ sobre ventas.

e) El porcentaje de empresas industriales que han registrado patentes, diseños industriales y derechos de autor es mayor que el sector de servicios, salvo en el registro de marcas donde es ligeramente mayor el porcentaje de este último sector.
2) Se encontraron algunos resultados en actividades donde el sector de servicios tiene una mayor intensidad en el proceso de innovación como son los siguientes.

a) El mayor porcentaje de empresas que han realizado innovaciones en la organización están en el sector de servicios que en el industrial en las tres variables consideradas, gestión de la información (68.2/60.6\%), cambios a nivel de la organización (61.4/52.1\%) y su relación con otras empresas $(41.9 / 33.8 \%)$. Este resultado es congruente con el hecho de que las empresas de servicios se orientan más a las innovaciones no tecnológicas.

b) Referente a los apoyos financieros, se encontró que tanto regional como federal, son las empresas de servicios que reciben un mayor apoyo financiero, $9.1 \%$ de las empresas del sector de servicios y $5.6 \%$ del sector industrial los reciben de instituciones regionales $\mathrm{y}$ $15.9 \%$ de las empresas de servicios y $9.9 \%$ del sector 
industrial los reciben de instituciones federales.

c) Se encontró que el esfuerzo relativo de la $\mathrm{I}+\mathrm{D}$ contratada es ligeramente mayor en el caso de las empresas de servicios (2.02/1.8\%), lo cual se puede deber a la ausencia de infraestructura de I+D propia.

3) En cuanto a las relaciones significativas encontradas se encontró.

a) Referente a las alianzas con otras organizaciones para la realización de innovaciones, se encontró una asociación significativa entre la realización de alianzas y el sector, que muestra que son las empresas de la industria las que emplean mayormente esta estrategia para la realización de innovaciones, tal como se muestra en el cuadro 9, con la prueba de la $C h i$ cuadrada.

b) En el sector industrial se encontraron asociaciones significativas entre el registro de patentes y los otros tres registros de propiedad intelectual considerados, tal como se muestra en el cuadro 7 la prueba de $C h i$ cuadrada.

c) Igualmente, se encontró en el sector industrial una asociación significativa entre el registro de patentes y la realización de alianzas con otras organizaciones para la innovación, tal como se muestra el cuadro 8 , la prueba de Chi cuadrada.

Estos resultados permitieron probar la hipótesis central que establece que las empresas del sector industrial, dada que la mayor parte de sus innovaciones son de tipo tecnológico, requieren una mayor inversión en $\mathrm{I}+\mathrm{D}$, registro de patentes y alianzas que las empresas en el sector de servicios cuya mayor parte de sus innovaciones son de tipo no tecnológico.

Como conclusión general se puede decir que este estudio aporta elementos que permiten una mayor comprensión sobre las diferencias en el proceso de innovación de las empresas en los sectores de servicios y de la industria en empresas pequeñas y medianas en un país en desarrollo, encontrándose diferencias significativas en las actividades de las innovaciones tecnológicas propias del sector industrial y no tecnológicas propias del sector de servicios.

\section{REFERENCIAS BIBLIOGRÁFICAS}

Aboal, D., \& Garda, P. (2016). Technological and nontechnological innovation and productivity in services vis-à-vis manufacturing sectors. Economics of Innovation \& New Technology, 25(5), 435-454. doi: 10.10 $80 / 10438599.2015 .1073$ 478.

Carrasco, F. C. (2015). Structures formed by innovative activities beyond the traditional separation between manufacturing and services: identification of patterns of innovation. Revista De Economía Mundial, (40), 23-45.

Casas, R. (2004). Conocimiento, tecnología y desarrollo en América Latina. Revista Mexicana de Sociologia, 66, 255-277.

Chesbrough, H. W. (2003). Open Innovation: The new imperative for creating and profiting from technology. Boston: Harvard Business School Press. 
Dandurand, L. (2005). Réflexion autour du concept d'innovation sociale, approche historique et comparative. Revue française d'administration publique, 115(3), 377 $382 . \quad$ doi:10.3917/ rfap.115.0377.

D’Ippolito, B., \& Timpano, F. (2016). The Role of NonTechnological Innovations in Services: The Case of Food Retailing. Creativity \& Innovation $\mathrm{Ma}$ nagement, 25(1), 73-89. doi:10.1111/caim.12156.

Drucker, P. (1984). Innovation and Entrepreneurship. Practice and Principles. Nueva York: Harper \& Row.

Escorsa P. y Valls, P. (2009). Tecnología e innovación en la empresa. Barcelona: UPC.

Etzkowitz, H. (2008). Triple Helix: University-industrygovernment innovation in action. Nueva York y Londres: Routledge.

Eurostat (2005). Manuel d'Oslo: Principes directeurs pour le recueil et l'interprétation des données sur linnovation. OECD Publishing.
Freeman, C., \& Freeman, C. (1975). La teoría económica de la innovación industrial (Núm. 04; HD45, F7.).

Friedman, T. (2005). The World Is Flat: A Brief History of the Twenty-first Century. Nueva York: Farrar, Straus and Giroux.

Gianiodis, T., Ettlie E., y Urbina, J. (2014). Open service innovation in the global banking industry: Inside-out versus outsidein strategies. Academy of Management Perspectives, 28(1),76-91. doi:10.5465/ amp.2012.0126.

Gobble, M. (2010). The 2009 European Innovation Scoreboard: eu lags us and Japan while China closing gap with eu. Research Technology Management, 53(5), 2-4.

Jacobsson, S. y Bergek A. (2006). A framework for guiding policy makers intervening in emerging innovation systems in "catching up" countries. European Journal of Development Research, 8(4), 21.

Jasso, J. y Torres A. (2012). Innovación y crisis en empresas globales en países en desarrollo, en Innovación y crisis, trayectorias y respuestas de empresas y sectores. México: uam, Miguel Ángel Porrúa.

Leiponen, A. (2012). The benefits of $R \& D$ and breadth in innovation strategies: a comparison of Finnish service and manufacturing firms. Industrial \& Corporate Change, 21(5), 12551281.

ocde (2011), OECD Science, Technology and Industry Scoreboard 2011, Innovation and Growth in Knowledge Economies, Paris: ocde.

Ollivier, J. (2007). Proveeduría nacional a la industria maquiladora en México: Un reto tecnológico. Frontera norte, 19(38), 191218. (2009). Diferencias en el proceso de innovación en empresas pequeñas y medianas de la industria manufacturera de la ciudad de Chihuahua. Contaduría y Administración. México: unam, 227, pp. 9-28.

Pavitt, K. (1984). Sectoral patterns of technical change: towards a taxonomy 
and a theory. Research poli$c y, 13(6), 343-373$.

Pedroza, A., (2013). Innovación y tecnología en la empresa. Claves para adelantarse al futuro. Guadalajara, México: Instituto Tecnológico de Estudios Superiores.

Rothwelly W. Zegveld (1982). Innovation and Small and Medium Sized Enterprises. London: Pinter.

Santiago, F., De Fuentes, C., Dutrénit, G., y Gras, N. (2017). What hinders innovation performance of services and manufacturing firms in Mexico? Economics of Innovation \& $\mathrm{New} \mathrm{Te}$ chnology, 26(3), 247-268. doi: 10.1080/10438599. 2016.1181297.

Schumpeter, J. (1934). The Theory of Economic Development. Cambridge: Cambridge University Press.

Teixeira, A. y Bezerra, L. (2016). Innovation performance in service companies and kibs vis-à-vis manufacturing: the relevance of $a b-$ sorptive capacity and openness. Revista Brasileira De Gestão De Negócios, 18(59), 43-66. doi:10.7819/rbgn. v18i59.2215.
Visnjic Kastalli, I., Van Looy, B., y Neely, A. (2013). Steering Manufacturing Firms Towards Service Business Model Innovation. California Management Review, 56(1), 100-123.

Von Hippel, E. (2005), Democratizing Innovation, Cambridge M., London, MIT PRESS. 